\title{
Aminoácidos Digestíveis Verdadeiros da Soja e Subprodutos, Determinados com Galos Cecectomizados ${ }^{1}$
}

\author{
Paulo Borges Rodrigues ${ }^{2}$, Horacio Santiago Rostagno ${ }^{3}$, Luiz Fernando Teixeira Albino ${ }^{3}$, \\ Paulo Cezar Gomes ${ }^{3}$, Rodrigo Toledo Santana ${ }^{4}$, Ricardo Vianna Nunes ${ }^{4}$
}

RESUMO - Os coeficientes de digestibilidade e o conteúdo de aminoácidos digestíveis verdadeiros da soja e subprodutos foram determinados, utilizando-se galos cecectomizados, onde cada um dos 8 alimentos testados (amostras de soja e subprodutos) foram fornecidos a seis galos (15 g pela manhã e $15 \mathrm{~g}$ à tarde, após jejum de 24 horas) e seis galos foram mantidos em jejum, para determinação das perdas endógenas e metabólicas. Após obtidos os coeficientes de digestibilidade e calculados os aminoácidos digestíveis, ajustou-se equações para predizer a composição da soja e seus subprodutos em lisina (LIS), metionina (MET), metionina + cistina $(M+C)$, treonina (TRE) e arginina ( $A R G)$, totais e digestíveis, em função da composição em proteína bruta (PB), fibra bruta (FB), extrato etéreo (EE) e matéria mineral (MM). Os coeficientes médios de digestibilidade dos aminoácidos (essenciais e não essenciais, respectivamente) dos farelos de soja 1, 2, 3, 4 e texturizado, sojas integral Jet Sploder, integral tostada e micronizada foram 92,78 e 90,76; 92,66 e 89,70; 93,75 e 91,26; 93,32 e 90,12; 91,55 e 89,08; 94,80 e 92,34; 94,61 e 91,$47 ; 93,20$ e 90,47 \%. As equações foram bem ajustadas, cujos valores de $\mathrm{R}^{2}$ foram superiores a $91 \%$ e a PB apresentou alta correlação positiva, onde as equações LIS $=-0,32161+0,06731 \mathrm{~PB}\left(\mathrm{R}^{2}=0,99\right) ;$ MET $=0,02263+0,01323 \mathrm{~PB}\left(\mathrm{R}^{2}=0,96\right) ;$ $\mathrm{M}+\mathrm{C}=0,078949+0,02563 \mathrm{~PB}\left(\mathrm{R}^{2}=0,94\right) ; \mathrm{TRE}=0,20930+0,03533 \mathrm{~PB}\left(\mathrm{R}^{2}=0,95\right) ; \mathrm{ARG}=-0,39589+0,08022 \mathrm{~PB}\left(\mathrm{R}^{2}=\right.$ $0,98)$ e LIS $=-0,29472+0,06319 \mathrm{~PB}\left(\mathrm{R}^{2}=0,98\right) ; \mathrm{MET}=0,02884+0,01228 \mathrm{~PB}\left(\mathrm{R}^{2}=0,93\right) ; \mathrm{M}+\mathrm{C}=0,08974+0,0222 \mathrm{~PB}\left(\mathrm{R}^{2}\right.$ $=0,91) ; \mathrm{TRE}=0,15813+0,03235 \mathrm{~PB}\left(\mathrm{R}^{2}=0,95\right)$ e $\mathrm{ARG}=-0,32129+0,07681 \mathrm{~PB}\left(\mathrm{R}^{2}=0,96\right)$, podem predizer o conteúdo em aminoácidos totais e digestíveis da soja e subprodutos, respectivamente.

Palavras-chave: aminoácidos digestíveis, galos cecectomizados, soja, equações de predição

\section{True Digestible Amino Acids of Soybean and Soybean By-products, Determined with Cecectomized Cockerels}

\begin{abstract}
S - The digestibility coefficients and the true digestible amino acids content of soybean and soybean by-products were determined using cecectomized adult cockerels. Each one of the 8 feedstuffs evaluated was fed to six cockerels, consuming $15 \mathrm{~g}$ at 8:00 A.M. and $15 \mathrm{~g}$ at 16:00h P.M., after 24 hours fasting. Simultaneously, six cockerels were fasted to determine metabolic and endogenous losses. After determination of the digestibility coefficients and calculation of the digestible amino acids, prediction equations were simulated to predict the soybean and soybean byproducts total and digestible composition in lysine (LYS), methionine (MET), methionine plus cystine (Met + Cys), threonine (THR) and arginine (ARG), as a function of the chemical composition (crude protein - CP, crude fiber - CF, ether extract - EE and ash). The average amino acids digestibility coefficient (essential and non essential) of soybean meal samples (1, 2, 3, 4 and texturized), full fat soybean Jet Sploder, toasted and micronized were 92,78 and 90,76; 92,66 and 89,70; 93,75 and 91,26; 93,32 and 90,$12 ; 91,55$ and 89,$08 ; 94,80$ and 92,$34 ; 94,61$ and 91,$47 ; 93,20$ and 90,47\%, respetively. The prediction equations were adjusted with higher $\mathrm{R}^{2}$ values higher than $91 \%$, and crude protein showed high positive correlation. The equations $\mathrm{LYS}$ $=-0.32161+0.06731 \mathrm{~PB}\left(\mathrm{R}^{2}=0.99\right) ; \mathrm{MET}=0.02263+0.01323 \mathrm{~PB}\left(\mathrm{R}^{2}=0.96\right) ; \mathrm{Met}+\mathrm{Cys}=0.078949+0.02563 \mathrm{~PB}\left(\mathrm{R}^{2}=\right.$ $0.94) ;$ THR $=0.20930+0.03533 \mathrm{~PB}\left(\mathrm{R}^{2}=0.95\right) ; \mathrm{ARG}=-0.39589+0.08022 \mathrm{~PB}\left(\mathrm{R}^{2}=0.98\right)$ e $\mathrm{LYS}=-0.29472+0.06319 \mathrm{~PB}$ $\left(\mathrm{R}^{2}=0.98\right) ;$ MET $=0.02884+0.01228 \mathrm{~PB}\left(\mathrm{R}^{2}=0.93\right) ;$ Met $+\mathrm{Cys}=0.08974+0.02220 \mathrm{~PB}\left(\mathrm{R}^{2}=0.91\right) ; \mathrm{THR}=0.15813+$ $0.03235 \mathrm{~PB}\left(\mathrm{R}^{2}=0.95\right)$ e $\mathrm{ARG}=-0.32129+0.07681 \mathrm{~PB}\left(\mathrm{R}^{2}=0.96\right)$, can be used to predict the total and digestible amino acids content of soybean and byproducts, respectively.
\end{abstract}

Key Words: digestible amino acids, cecectomized cockerels, soybean, prediction equations

\footnotetext{
${ }_{1}^{1}$ Parte da tese de doutorado do primeiro autor.

2 Professor do Departamento de Zootecnia da UFLA. E.mail: pborges@ufla.br

${ }^{3}$ Professores do Departamento de Zootecnia da UFV. E.mail: rostagno@ufv.br

${ }^{4}$ Alunos de doutorado do Departamento de Zootecnia da UFV.
} 


\section{Introdução}

A formulação e o balanceamento de ração consistem na mistura de vários alimentos com o objetivo de atender, adequadamente, às exigências nutricionais dos animais, em cada fase de criação, para que os mesmos possam expressar todo seu potencial genético. Para que haja maior precisão neste processo, há a necessidade do conhecimento da composição nutricional dos alimentos, bem como suas limitações de uso (Albino \& Silva, 1996). A soja, seja na forma de grãos ou farelo, é destacada na literatura como importante matéria prima na formulação de rações, devido seu elevado valor nutritivo. De acordo com Dale (1997), vários estudos vem sendo realizados visando obter um máximo aproveitamento de suas propriedades nutricionais já que, na maioria das vezes, o farelo de soja é responsável por aproximadamente $70 \%$ da suplementação proteica nas rações.

A maior parte dos aminoácidos ingeridos pelas aves está na forma de proteína que, ao serem digeridas, liberam estes para que sejam absorvidos (Albino et al., 1992). No entanto, os aminoácidos não são totalmente disponíveis para a absorção e, desta forma, o conhecimento dos coeficientes de digestibilidade verdadeira destes, nos diferentes alimentos, pode ser imprescindível para que os nutricionistas maximizem seu aproveitamento, aumentando a produtividade e retorno econômico da produção (Fischer Jr. et al., 1998). Assim, o interesse pela digestibilidade dos aminoácidos nos alimentos é crescente e, segundo Rostagno et al. (1999), já é uma realidade o fato de que os aminoácidos dos alimentos devem ser expressos na forma de aminoácidos digestíveis. Também a possibilidade de se utilizar equações que possam predizer a composição dos alimentos em aminoácidos, a partir de sua composição química tem sido muito destacada atualmente. O NRC (1998) traz uma série de equações estabelecidas para estimar a composição de alguns aminoácidos essenciais totais.

Dessa forma, o presente trabalho foi conduzido com o objetivo de determinar os coeficientes de digestibilidade dos aminoácidos da soja integral e subprodutos e o conteúdo de aminoácidos digestíveis verdadeiros destes alimentos. Posteriormente, utilizando a composicão deste grupo de alimentos, em proteína bruta, fibra bruta, extrato etéreo e matéria mineral, ajustaram-se equações para predizer o conteúdo de alguns aminoácidos essenciais totais e digestíveis.

R. Bras. Zootec., v.31, n.2, p.970-981, 2002 (suplemento)

\section{Material e Métodos}

O ensaio de digestibilidade foi conduzido no Laboratório Animal do DZO/UFV, utilizando-se o método de alimentação forçada, também conhecido como alimentação precisa (Sibbald, 1979), com galos Leghorne adultos cecectomizados, com 21 meses de idade e peso médio de $2132 \pm 257 \mathrm{~g}$. Os galos foram cecectomizados por meio de laparotomia abdominal e anestesia local, conforme a metodologia descrita por Pupa et al. (1998). As temperaturas mínimas e máximas médias registradas no período experimental fo$\operatorname{ram} 22,9 \pm 1,1^{\circ} \mathrm{C}$ e $28,9 \pm 3,1^{\circ} \mathrm{C}$, respectivamente.

Foram determinados os coeficientes de digestibilidade verdadeira de oito alimentos, sendo quatro marcas comerciais de farelo de soja (denominados farelos de soja 1, 2, 3 e 4), farelo de soja texturizado, soja integral "Jet Sploder", soja integral tostada e soja micronizada. Os grãos de soja (integral, Jet Sploder e micronizada) e farelo texturizado foram processados conforme descrição de Rodrigues (2000).

Cada um dos alimentos foi fornecido a seis galos, sendo um galo por unidade experimental e, simultaneamente, seis galos foram mantidos em jejum para determinação das perdas endógenas e metabólicas. Antes do período experimental, os galos foram alojados nas baterias e passaram por um período de adaptação, recebendo alimentação em dois turnos de 1 hora, às 8 e 16 h, visando à dilatação do papo. Em seguida, foram mantidos em jejum por 24 horas com o objetivo de esvaziar o trato digestivo e, então, forçados a ingerir $30 \mathrm{~g}$ do alimento teste, por meio de um funil-sonda introduzido via esôfago até o papo, sendo fornecidas $15 \mathrm{~g}$ dos alimentos às 8 e $15 \mathrm{~g}$ às $16 \mathrm{~h}$, para evitar regurgitações. As bandejas sob as gaiolas foram revestidas com plástico, e a coleta de excretas realizada às 8 e 16 horas, por um período de 56 horas, após iniciado o fornecimento dos alimentos. As excretas foram coletadas e acondicionadas em freezer até o final do experimento, quando foram descongeladas, devidamente quantificadas, homogeneizadas e secas em estufa de ventilação forçada a $55{ }^{\circ} \mathrm{C}$ por um período de 72 horas. Posteriormente as amostras dos alimentos foram analisadas em matéria seca e nitrogênio, através das metodologias descritas por Silva (1990) e o conteúdo em aminoácidos determinados no Laboratório da Eurolysine (Amiens - França).

Os coeficientes de digestibilidade de cada aminoácido dos alimentos, calculados pela fórmula apresentada abaixo, foram submetidos à análise esta- 
tística e aqueles cuja análise de variância apresentaram efeito significativo $(\mathrm{P}<0,05)$ foram comparados pelo teste de agrupamento de Scott-Knott através do pacote SAEG versão 5.0 (Sistema para Análises Estatísticas - UFV, 1992).

CDVaa=aminoácido (aa) ingerido-aa excretado + aadafraçãoendógena aa ingerido (em gramas)

Posteriormente, após calculados o conteúdo de aminoácidos digestíveis verdadeiros de cada alimento, foram estimadas equações para predizer a composição em alguns aminoácidos totais e digestíveis (lisina, metionina, metionina + cistina, treonina e arginina) deste grupo de alimentos, por meio de regressões lineares simples e múltiplas, pelo Método de Eliminação Indireta ou Backward, utilizando-se o pacote SAEG, mencionado anteriormente. Utilizaram-se na predição das equações os valores de proteína bruta (PB), fibra bruta (FB), extrato etéreo (EE) e matéria mineral (MM). Para obter equações de maior precisão, foram adotados o teste $\mathrm{T}$ e uma significância de 5\% de probabilidade para cada variável componente do modelo, sendo consideradas somente as equações em que todas as variáveis independentes apresentassem significância.

\section{Resultados e Discussão}

A composição em aminoácidos essenciais e não essenciais dos alimentos estudados encontram-se apresentadas nas Tabelas 1 e 2, respectivamente, e mostra uma variação em relação aos valores apresentados na literatura, com alguns aminoácidos acima, abaixo ou semelhante aos observados no presente trabalho. O teor médio de lisina dos farelos de soja, por exemplo, foi 5,90; 8,12; 5,17 e 7,01\% inferior aos valores apresentados por Rostagno et al. (1983), Fischer Jr. (1997), Bellaver et al. (1998) e Dale (1999), respectivamente, sendo 7,54 e 4,32 \% superior aos apresentados por Albino et al. (1992) e Pupa (1995) e semelhante aos valores descritos por Rhône Poulenc (1993) e NRC (1994). Observação semelhante ocorre quando se comparam os resultados com as tabelas da Embrapa (1991), Degussa (1993) e NRC (1998).

A média da lisina das amostras de soja integral (Jet Sploder e tostada) diferiram quando comparada aos valores de Rhône Poulenc (1993), NRC (1994), Fischer Jr. (1997), e foi semelhante àquele descrito no NRC (1998). Dessa forma, nota-se que não só a lisina, mas

Tabela 1 - Conteúdo de aminoácidos essenciais totais da soja e subprodutos

Table 1 - Total essential amino acids contents of the soybean and by-products

\begin{tabular}{|c|c|c|c|c|c|c|c|c|c|c|c|c|}
\hline \multirow[b]{2}{*}{$\begin{array}{l}\text { Alimento } \\
\text { Feedstuff }\end{array}$} & \multicolumn{12}{|c|}{$\begin{array}{c}\text { Aminoácidos } 1,2 \\
\text { Amino acids }\end{array}$} \\
\hline & $\begin{array}{l}\text { MS } \\
D M \\
(\%) \\
\end{array}$ & $\begin{array}{l}\mathrm{PB} \\
C P \\
(\%) \\
\end{array}$ & $\begin{array}{l}\text { LIS } \\
\text { LYS } \\
(\%)\end{array}$ & $\begin{array}{c}\text { MET } \\
M E T \\
(\%)\end{array}$ & $\begin{array}{c}\mathrm{M}+\mathrm{C} \\
M e t+C y s \\
(\%)\end{array}$ & $\begin{array}{l}\text { TRE } \\
\text { THR } \\
(\%)\end{array}$ & $\begin{array}{c}\text { ARG } \\
A R G \\
(\%)\end{array}$ & $\begin{array}{l}\text { HIS } \\
H Y S \\
(\%)\end{array}$ & $\begin{array}{l}\text { ILE } \\
\text { ILE } \\
(\%)\end{array}$ & $\begin{array}{c}\mathrm{LEU} \\
L E U \\
(\%)\end{array}$ & $\begin{array}{l}\text { FEN } \\
P H E \\
(\%)\end{array}$ & $\begin{array}{l}\text { VAL } \\
\text { VAL } \\
(\%)\end{array}$ \\
\hline $\begin{array}{l}\text { Farelo de soja } 1 \\
\text { Soybean meal } 1\end{array}$ & 89,17 & 44,09 & 2,71 & 0,61 & 1,23 & 1,77 & 3,16 & 1,12 & 2,06 & 3,37 & 2,23 & 2,10 \\
\hline $\begin{array}{l}\text { Farelo de soja } 2 \\
\text { Soybean meal } 2\end{array}$ & 89,37 & 43,43 & 2,66 & 0,61 & 1,20 & 1,74 & 3,09 & 1,11 & 1,92 & 3,37 & 2,15 & 1,99 \\
\hline $\begin{array}{l}\text { Farelo de soja } 3 \\
\text { Soybean meal } 3\end{array}$ & 89,66 & 44,51 & 2,65 & 0,60 & 1,19 & 1,70 & 3,19 & 1,09 & 1,98 & 3,32 & 2,20 & 2,12 \\
\hline $\begin{array}{l}\text { Farelo de soja } 4 \\
\text { Soybean meal } 4\end{array}$ & 89,35 & 46,43 & 2,83 & 0,62 & 1,21 & 1,80 & 3,41 & 1,19 & 2,18 & 3,57 & 2,40 & 2,25 \\
\hline $\begin{array}{l}\text { Farelo texturizado } \\
\text { Texturized soybean meal }\end{array}$ & 93,65 & 49,30 & 3,03 & 0,68 & 1,37 & 1,98 & 3,61 & 1,26 & 2,19 & 3,73 & 2,57 & 2,43 \\
\hline $\begin{array}{l}\text { Soja "Jet Sploder" } \\
\text { "Jet Sploder" soybean }\end{array}$ & 90,18 & 36,96 & 2,15 & 0,50 & 1,01 & 1,49 & 2,52 & 0,91 & 1,74 & 2,89 & 1,88 & 1,75 \\
\hline $\begin{array}{l}\text { Soja integral tostada } \\
\text { Toasted integral soybean }\end{array}$ & 92,35 & 37,97 & 2,29 & 0,54 & 1,06 & 1,57 & 2,74 & 0,98 & 1,87 & 2,99 & 2,01 & 1,94 \\
\hline $\begin{array}{l}\text { Soja micronizada } \\
\text { Micronized soybean }\end{array}$ & 93,48 & 38,53 & 2,31 & 0,52 & 1,05 & 1,53 & 2,78 & 0,99 & 1,86 & 3,02 & 2,02 & 1,98 \\
\hline
\end{tabular}

${ }^{1}$ Análises realizadas no Laboratório da Eurolysine (Amiens - França).

2 MS (matéria seca), PB (proteína bruta), LIS (lisina), MET (metionina), M+C (metionina + cistina), TRE (treonina), ARG (arginina), HIS (histidina), ILE (isoleucina), LEU (leucina), FEN (fenilalanina), VAL (valina).

${ }^{1}$ Analyzed in the Eurolysine Laboratory (Amiens - France).

${ }^{2}$ DM (dry matter), CP (crude protein), LYS (lisina), MET (methionine), Met+Cys (methionine + cystine), THR (treonine), ARG (arginine), HIS (hystidine), ILE (isoleucyne), LEU (leucyne), PHE (phenylalanine), VAL (valyne).

\section{R. Bras. Zootec., v.31, n.2, p.970-981, 2002 (suplemento)}


Tabela 2 - Conteúdo de aminoácidos não essenciais totais da soja e subprodutos Table 2 - Total non essential amino acids content of the soybean and by-products

\begin{tabular}{|c|c|c|c|c|c|c|c|c|c|}
\hline \multirow{3}{*}{$\begin{array}{l}\text { Alimento } \\
\text { Feedstuff }\end{array}$} & \multicolumn{9}{|c|}{$\begin{array}{l}\text { Aminoácidos } \\
\text { Amino acids }\end{array}$} \\
\hline & MS & $\mathrm{PB}$ & CIS & ALA & ASP & GLU & GLI & SER & TIR \\
\hline & $\begin{array}{l}D M \\
(\%)\end{array}$ & $\begin{array}{l}C P \\
(\%)\end{array}$ & $\begin{array}{l}C Y S \\
(\%)\end{array}$ & $\begin{array}{l}A L A \\
(\%)\end{array}$ & $\begin{array}{l}A S P \\
(\%)\end{array}$ & $\begin{array}{l}G L U \\
(\%)\end{array}$ & $\begin{array}{l}G L Y \\
(\%)\end{array}$ & $\begin{array}{l}\text { SER } \\
(\%)\end{array}$ & $\begin{array}{l}\text { TYR } \\
(\%)\end{array}$ \\
\hline Farelo de soja 1 & 89,17 & 44,09 & 0,62 & 1,83 & 5,11 & 8,16 & 1,86 & 2,19 & 1,43 \\
\hline $\begin{array}{l}\text { Soybean meal } 1 \\
\text { Farelo de soja } 2 \\
\text { Soybean meal } 2\end{array}$ & 89,37 & 43,43 & 0,59 & 1,87 & 5,15 & 8,21 & 1,92 & 2,29 & 1,50 \\
\hline $\begin{array}{l}\text { Farelo de soja } 3 \\
\text { Soybean meal } 3\end{array}$ & 89,66 & 44,51 & 0,60 & 1,83 & 5,12 & 7,98 & 1,85 & 2,16 & 1,46 \\
\hline $\begin{array}{l}\text { Farelo de soja } 4 \\
\text { Soybean meal } 4\end{array}$ & 89,35 & 46,43 & 0,58 & 1,88 & 5,29 & 8,54 & 1,91 & 2,25 & 1,60 \\
\hline $\begin{array}{l}\text { Farelo texturizado } \\
\text { Texturized soybean meal }\end{array}$ & 93,65 & 49,30 & 0,69 & 2,03 & 5,75 & 9,03 & 2,03 & 2,45 & 1,70 \\
\hline $\begin{array}{l}\text { Soja "Jet Sploder" } \\
\text { "Jet Sploder" soybean }\end{array}$ & 90,18 & 36,96 & 0,51 & 1,52 & 4,30 & 6,83 & 1,59 & 1,87 & 1,33 \\
\hline $\begin{array}{l}\text { Soja integral tostada } \\
\text { Toasted integral soybean }\end{array}$ & 92,35 & 37,97 & 0,51 & 1,60 & 4,44 & 7,06 & 1,65 & 1,87 & 1,33 \\
\hline $\begin{array}{l}\text { Soja micronizada } \\
\text { Micronized soybean }\end{array}$ & 93,48 & 38,53 & 0,54 & 1,63 & 4,50 & 7,12 & 1,66 & 1,85 & 1,37 \\
\hline
\end{tabular}

também os demais aminoácidos essenciais variam em composição, dentro de amostras, como observado para o farelo de soja no presente trabalho e também por Pupa (1995), e em comparação com a literatura. Estas observações reforçam as colocações de Albino et al. (1987) de que a composição dos alimentos é influenciada pela variação nos solos e climas em que são cultivados, variedades e pelo processamento a que são submetidos. O farelo de soja texturizado apresentou uma composição em todos aminoácidos essenciais e não essenciais superior aos demais alimentos do grupo estudados, possivelmente devido ao seu maior conteúdo em PB, quando comparado aos demais alimentos, sendo o inverso observado para a soja integral Jet Sploder, com exceção da cistina, serina e tirosina, os quais foram semelhantes à soja integral tostada. Por outro lado, o conteúdo de aminoácidos da soja micronizada foi bem similar ao da integral tostada e ligeiramente superior à soja integral Jet Sploder.

Nas Tabelas 3 e 4 encontram-se as médias dos coeficientes de digestibilidade dos aminoácidos essenciais e dos não essenciais, respectivamente. Com exceção da metionina + cistina, treonina e valina, que apresentaram alguns coeficientes de digestibilidade abaixo de $90 \%$, os demais aminoácidos essenciais tiveram digestibilidade superior a $91 \%$, onde os valores observados para a arginina foram os mais elevados, em todos os alimentos estudados. Apesar da metionina + cistina ter apresentado digestibilidades inferiores, os valores obtidos para a metionina foram, exceto para a soja integral Jet Sploder (91,87\%) superiores a $93 \%$. A cistina foi, dos aminoácidos não essenciais, a que teve coeficientes de digestibilidade mais baixos, variando de $80,72 \%$ (soja integral Jet Sploder) a $85,86 \%$ (soja integral tostada), seguida da alanina, cujos coeficientes variaram de 83,96 a 89,46\%, para a amostra de farelo de soja 4 e soja integral tostada, respectivamente. Os demais aminoácidos não essenciais analisados tiveram digestibilidades verdadeiras superiores a $90 \%$.

Esta baixa digestibilidade da cistina possivelmente explica os coeficientes mais baixos obtidos para metionina + cistina, quando comparados aos da metionina. Comportamento semelhante pode ser observado nos resultados de Pupa (1995) e Fischer Jr. et al. (1998), sugerindo que, independentemente do alimento avaliado, a cistina tem digestibilidade inferior à metionina, resultando em menores coeficientes 


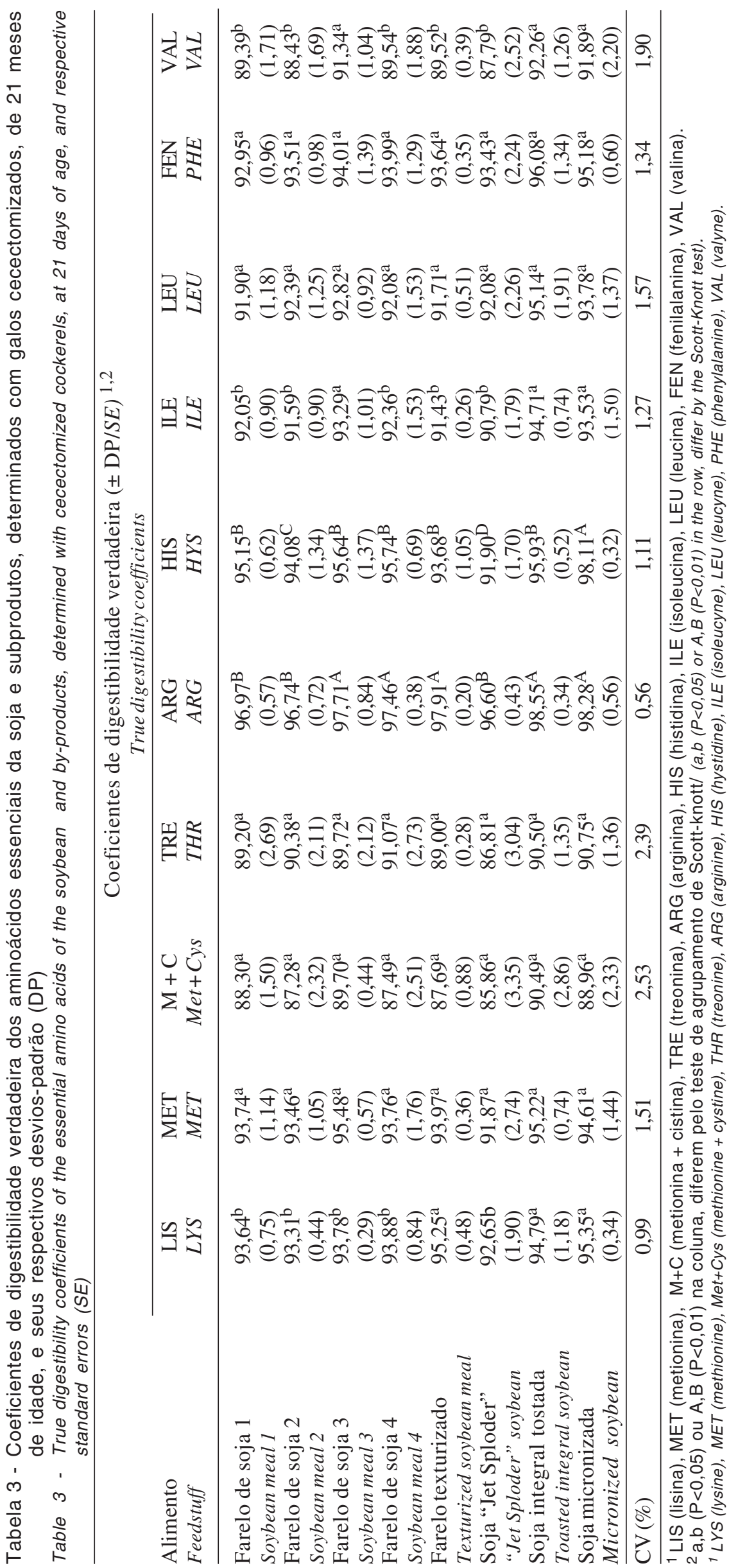


Tabela 4 - Coeficientes de digestibilidade verdadeira dos aminoácidos não essenciais da soja e subprodutos, determinados com galos cecectomizados, de 21 meses de idade, e seus respectivos desvios-padrão (DP).

Table 4 - True digestibility coefficients of the non essential amino acids of the soybean and by-products, determined with cecectomized cockerels, at 21 days of age, and respective standard errors (SE)

\begin{tabular}{|c|c|c|c|c|c|c|}
\hline \multirow{3}{*}{$\begin{array}{l}\text { Alimento } \\
\text { Feedstuff }\end{array}$} & \multicolumn{6}{|c|}{$\begin{array}{c}\text { Coeficientes de digestibilidade verdadeira }( \pm \mathrm{DP} / S E)^{1,2} \\
\text { True digestibility coefficients }\end{array}$} \\
\hline & CIS & ALA & ASP & GLU & SER & TIR \\
\hline & $C Y S$ & $A L A$ & $A S P$ & $G L U$ & SER & $T Y R$ \\
\hline Farelo de soja 1 & $82,94^{\mathrm{a}}(2,15)$ & $86,70^{\mathrm{a}}(1,89)$ & $92,83^{\mathrm{a}}(0,53)$ & $95,62^{\mathrm{a}}(0,86)$ & $91,84^{\mathrm{a}}(0,96)$ & $94,65^{\mathrm{a}}(1,44)$ \\
\hline $\begin{array}{l}\text { Soybean meal } 1 \\
\text { Farelo de soja } 2 \\
\text { Soybean meal } 2\end{array}$ & $81,14^{\mathrm{a}}(4,14)$ & $84,86^{\mathrm{b}}(0,65)$ & $91,41^{\mathrm{b}}(0,55)$ & $94,59^{\mathrm{b}}(0,46)$ & $91,97^{\mathrm{a}}(1,55)$ & $94,23^{\mathrm{a}}(1,26)$ \\
\hline $\begin{array}{l}\text { Farelo de soja } 3 \\
\text { Soybean meal } 3\end{array}$ & $83,92^{\mathrm{a}}(0,53)$ & $87,11^{\mathrm{a}}(1,20)$ & $92,29^{\mathrm{a}}(0,61)$ & $95,62^{\mathrm{a}}(0,67)$ & $93,57^{\mathrm{a}}(1,65)$ & $95,07^{\mathrm{a}}(0,72)$ \\
\hline $\begin{array}{l}\text { Farelo de soja } 4 \\
\text { Soybean meal } 4\end{array}$ & $80,96^{\mathrm{a}}(2,80)$ & $83,96^{\mathrm{b}}(2,90)$ & $93,24^{\mathrm{a}}(0,24)$ & $95,03^{\mathrm{b}}(0,51)$ & $92,55^{\mathrm{a}}(1,53)$ & $94,98^{\mathrm{a}}(1,01)$ \\
\hline $\begin{array}{l}\text { Farelo texturizado } \\
\text { Texturized soybean meal }\end{array}$ & $81,51^{\mathrm{a}}(2,08)$ & $84,21^{\mathrm{b}}(0,35)$ & $92,56^{\mathrm{a}}(0,78)$ & $96,17^{\mathrm{a}}(0,13)$ & $92,51^{\mathrm{a}}(0,59)$ & $95,89^{\mathrm{a}}(0,60)$ \\
\hline $\begin{array}{l}\text { Soja "Jet Sploder" } \\
\text { "Jet Sploder" soybean }\end{array}$ & $80,72^{\mathrm{a}}(1,97)$ & $84,15^{\mathrm{b}}(2,37)$ & $90,85^{\mathrm{b}}(1,13)$ & $94,04^{\mathrm{b}}(1,53)$ & $90,10^{\mathrm{a}}(1,58)$ & $94,62^{\mathrm{a}}(1,06)$ \\
\hline $\begin{array}{l}\text { Soja integral tostada } \\
\text { Toasted integral soybean }\end{array}$ & $85,86^{\mathrm{a}}(4,60)$ & $89,46^{\mathrm{a}}(2,79)$ & $92,64^{\mathrm{a}}(0,95)$ & $96,11^{\mathrm{a}}(0,83)$ & $93,75^{\mathrm{a}}(0,99)$ & $96,22^{\mathrm{a}}(0,44)$ \\
\hline $\begin{array}{l}\text { Soja micronizada } \\
\text { Micronized soybean }\end{array}$ & $83,50^{\mathrm{a}}(3,19)$ & $87,57^{\mathrm{a}}(1,47)$ & $91,81^{\mathrm{b}}(1,08)$ & $95,71^{\mathrm{a}}(0,49)$ & $93,38^{\mathrm{a}}(2,01)$ & $96,83^{\mathrm{a}}(1,25)$ \\
\hline $\mathrm{CV}(\%)$ & 3,57 & 2,24 & 0,85 & 0,83 & 1,54 & 1,08 \\
\hline
\end{tabular}

${ }^{1}$ CIS (cistina), ALA (alanina), SER (serina), GLU (ácido glutâmico), ASP (ácido aspártico), TIR (tirosina).

${ }^{2} A, B$ na coluna, diferem pelo teste de agrupamento de Scott-knott $(P<0,01)(A, B$ in the row, differing by the Scott-Knott test).

${ }^{1}$ CYS (cystine), ALA (alanine), SER (serine), GLU (glutamic acid), ASP (aspartic acid), TYR (tyrosine).

de digestibilidade da metionina + cistina. Quando se compara os coeficientes de digestibilidade dos farelos de soja, obtidos no presente trabalho, com a literatura, pode-se observar uma variabilidade na maioria dos aminoácidos avaliados (Albino et al., 1992; Degussa, 1993; Rhône Poulenc, 1993; Pupa, 1995; Fischer Jr. et al., 1998). Estas variações observadas entre a literatura e mesmo entre os alimentos do presente trabalho estão, certamente, associadas ao processamento de calor ao qual estes alimentos são submetidos. Uma aplicação excessiva de calor pode reduzir a disponibilidade dos aminóacidos por mudanças fisico-químicas, como derivação da lisina, oxidação do enxofre na cisteína e metionina e ligações cruzadas em amidas e carboxilas (Araba \& Dale, 1990). O coeficiente médio de digestibilidade da lisina dos farelos de soja no presente ensaio foi, em média, 7,$64 ; 4,06 ; 2,53$ e $1,47 \%$ superior aos resultados obtidos por Degussa (1993); Rhône Poulenc (1993); Pupa (1995) e Bellaver et al. (1998), respectivamente. Estas variações, no entanto, possivelmente estão associadas a diferenças em análises laboratoriais e procedimentos experimentais, como limpeza do material a ser coletado e número de coletas por dia.
Quando se compara os alimentos estudados, notase que os coeficientes de digestibilidade da metionina, metionina + cistina, treonina, leucina e fenilalanina foram similares, quando agrupados pelo teste de Scott-Knott $(\mathrm{P}>0,05)$. Entretanto, a digestibilidade da lisina das 4 amostras de farelo de soja e soja integral Jet Sploder foram inferiores ao farelo de soja texturizado, sojas integral tostada e micronizada. A histidina da soja micronizada foi a que apresentou maior digestibilidade $(98,11 \%)$, sendo a da soja Jet Sploder menos digestível $(91,90 \%)$. Os coeficientes de digestibilidade da alanina e dos ácidos aspártico e glutâmico foram diferenciados entre os alimentos estudados e, quando se considera a digestibilidade média da soma dos não essenciais, não houve diferenças entre os alimentos, quando agrupados pelo teste de Scott-Knott $(\mathrm{P}>0,05)$.

A partir dos coeficientes de digestibilidade determinados para cada aminoácido no ensaio biológico, calculou-se o conteúdo de aminoácidos digestíveis dos alimentos os quais estão apresentados nas Tabelas 5 (essenciais) e 6 (não essenciais). Estes valores são de grande utilidade uma vez que, conforme Rostagno et al. (1999), já é realidade que nas 
formulações, os valores de aminoácidos devem ser expressos em termos de aminoácidos digestíveis. Assim, como observado para o conteúdo total de aminoácidos dos alimentos, houve certa variabilidade entre o conteúdo de aminoácidos digestíveis determinados no presente trabalho e aqueles relatados por Degussa (1993); Rhône Poulenc (1993); Pupa (1995) e Fischer Jr. et al. (1998), para o farelo de soja e entre os valores do presente trabalho e aqueles de Fischer Jr. et al. (1998) para a soja integral tostada, tanto essenciais como não essenciais. Esta diferença pode ser associada à variação observada, tanto no conteúdo total quanto nos coeficientes de digestibilidade determinados, o que pode, consequentemente, resultar em variáveis conteúdos de aminoácidos digestíveis.

Nas Tabelas 7 e 8 estão apresentadas as equações estabelecidas para predizer o conteúdo em lisina, metionina, metionina + cistina, treonina e arginina totais e digestíveis, respectivamente, em função da composição química (PB, FB, EE, MM) da soja integral e subprodutos e suas respectivas correlações com os aminoácidos. Nota-se que, com exceção da arginina, em que o EE teve participação significativa nas equações desenvolvidas, as demais equações somente foram ajustadas com a variável $\mathrm{PB}$, tanto para aminoácidos totais quanto digestíveis. Entretanto, os coeficientes de determinação foram elevados, explicando mais de $91 \%$ das variações no conteúdo de todos aminoácidos estudados. Os valores de $\mathrm{R}^{2}$ variaram de 0,91 (metionina + cistina digestível) a 0,99 (arginina total), mostrando que houve, no geral, bons ajustes.

A PB foi a variável que mais esteve correlacionada com os aminoácidos estudados, apresentando alta correlação positiva, cujos valores variaram de 97,16 a $99,35 \%$ para os totais e de 95,43 a $98,84 \%$ para metionina + cistina e lisina, totais e digestíveis, respectivamente. A MM, apesar de se correlacionar positivamente com os aminoácidos, com valores altos $(84,14$ a $88,77 \%$ para os totais e 84,06 a $88,53 \%$ para os digestíveis) não se ajustou significativamente em nenhum modelo. A FB também não participou das equações e, com exceção da metionina, que mostrou baixa correlação positiva com a fibra, os demais aminoácidos apresentaram correlação negativa inferior a $10 \%$. O EE se correlacionou negativamente,

Tabela 5 - Conteúdo de aminoácidos essenciais digestíveis da soja e subprodutos Table 5 - Digestible essential amino acids content of the soybean and by-products

\begin{tabular}{|c|c|c|c|c|c|c|c|c|c|c|c|c|}
\hline \multirow[b]{2}{*}{$\begin{array}{l}\text { Alimento } \\
\text { Feedstuff }\end{array}$} & \multicolumn{12}{|c|}{$\begin{array}{c}\text { Aminoácidos } \\
\text { Amino acids }\end{array}$} \\
\hline & $\begin{array}{l}\text { MS } \\
D M \\
(\%)\end{array}$ & $\begin{array}{l}\mathrm{PB} \\
C P \\
(\%)\end{array}$ & $\begin{array}{l}\text { LIS } \\
\text { LYS } \\
(\%)\end{array}$ & $\begin{array}{c}\text { MET } \\
M E T \\
(\%)\end{array}$ & $\begin{array}{c}\mathrm{M}+\mathrm{C} \\
M e t+C y s \\
(\%)\end{array}$ & $\begin{array}{l}\text { TRE } \\
\text { THR } \\
(\%)\end{array}$ & $\begin{array}{l}\mathrm{ARG} \\
A R G \\
(\%)\end{array}$ & $\begin{array}{l}\text { HIS } \\
\text { HYS } \\
(\%)\end{array}$ & $\begin{array}{l}\text { ILE } \\
I L E \\
(\%)\end{array}$ & $\begin{array}{l}\text { LEU } \\
\text { LEU } \\
(\%)\end{array}$ & $\begin{array}{l}\text { FEN } \\
P H E \\
(\%)\end{array}$ & $\begin{array}{l}\text { VAL } \\
\text { VAL } \\
(\%)\end{array}$ \\
\hline $\begin{array}{l}\text { Farelo de soja } 1 \\
\text { Soybean meal } 1\end{array}$ & 89,17 & 44,09 & 2,54 & 0,57 & 1,09 & 1,58 & 3,06 & 1,07 & 1,90 & 3,10 & 2,07 & 1,88 \\
\hline $\begin{array}{l}\text { Farelo de soja } 2 \\
\text { Soybean meal } 2\end{array}$ & 89,37 & 43,43 & 2,48 & 0,57 & 1,05 & 1,57 & 2,99 & 1,05 & 1,75 & 3,11 & 2,01 & 1,76 \\
\hline $\begin{array}{l}\text { Farelo de soja } 3 \\
\text { Soybean meal } 3\end{array}$ & 89,66 & 44,51 & 2,49 & 0,57 & 1,07 & 1,53 & 3,11 & 1,04 & 1,85 & 3,08 & 2,07 & 1,94 \\
\hline $\begin{array}{l}\text { Farelo de soja } 4 \\
\text { Soybean meal } 4\end{array}$ & 89,35 & 46,43 & 2,66 & 0,58 & 1,06 & 1,64 & 3,32 & 1,14 & 2,01 & 3,29 & 2,26 & 2,01 \\
\hline $\begin{array}{l}\text { Farelo texturizado } \\
\text { Texturized soybean meal }\end{array}$ & 93,65 & 49,30 & 2,88 & 0,64 & 1,20 & 1,76 & 3,53 & 1,21 & 2,00 & 3,42 & 2,41 & 2,18 \\
\hline $\begin{array}{l}\text { Soja "Jet Sploder" } \\
\text { "Jet Sploder" soybean }\end{array}$ & 90,18 & 36,96 & 2,00 & 0,46 & 0,86 & 1,30 & 2,43 & 0,84 & 1,58 & 2,66 & 1,76 & 1,54 \\
\hline $\begin{array}{l}\text { Soja integral tostada } \\
\text { Toasted integral soybean }\end{array}$ & 92,35 & 37,97 & 2,17 & 0,52 & 0,96 & 1,42 & 2,70 & 0,94 & 1,77 & 2,84 & 1,93 & 1,79 \\
\hline $\begin{array}{l}\text { Soja micronizada } \\
\text { Micronized soybean }\end{array}$ & 93,48 & 38,53 & 2,20 & 0,49 & 0,94 & 1,39 & 2,74 & 0,98 & 1,74 & 2,83 & 1,92 & 1,82 \\
\hline
\end{tabular}

${ }^{1}$ Valores calculados por meio dos coeficientes de digestibilidade determinados in vivo e conteúdo de aminoácidos totais dos alimentos.

${ }^{2}$ MS (matéria seca), PB (proteína bruta), LIS (lisina), MET (metionina), M+C (metionina + cistina), TRE (treonina), ARG (arginina), HIS (histidina), ILE (isoleucina), LEU (leucina), FEN (fenilalanina), VAL (valina), AAE (total dos aminoácidos essenciais).

1 Values calculated by the digestibility coefficients determined "in vivo" and total amino acids content of the feedstuffs.

2 DM (dry matter), CP (crude protein), LYS (lisina), MET (methionine), Met+Cys (methionine + cystine), THR (threonine), ARG (arginine), HIS (hystidine), ILE (isoleucyne), LEU (leucyne), PHE (phenylalanine), VAL (valyne).

\section{R. Bras. Zootec., v.31, n.2, p.970-981, 2002 (suplemento)}


Tabela 6 - Conteúdo de aminoácidos não essenciais digestíveis da soja e subprodutos Table 6 - Digestible non essential amino acids content of the soybean and by-products

\begin{tabular}{|c|c|c|c|c|c|c|}
\hline \multirow[b]{2}{*}{$\begin{array}{l}\text { Alimento } \\
\text { Feedstuff }\end{array}$} & \multicolumn{6}{|c|}{$\begin{array}{c}\text { Aminoácidos } \\
\text { Amino acids }\end{array}$} \\
\hline & $\begin{array}{l}\text { CIS } \\
C Y S \\
(\%)\end{array}$ & $\begin{array}{c}\text { ALA } \\
A L A \\
(\%)\end{array}$ & $\begin{array}{c}\text { ASP } \\
A S P \\
(\%)\end{array}$ & $\begin{array}{c}\text { GLU } \\
G L U \\
(\%)\end{array}$ & $\begin{array}{l}\text { SER } \\
\text { SER } \\
(\%)\end{array}$ & $\begin{array}{l}\text { TIR } \\
\text { TYR } \\
(\%)\end{array}$ \\
\hline $\begin{array}{l}\text { Farelo de soja } 1 \\
\text { Soybean meal } 1\end{array}$ & 0,51 & 1,59 & 4,74 & 7,80 & 2,01 & 1,35 \\
\hline $\begin{array}{l}\text { Farelo de soja } 2 \\
\text { Soybean meal } 2\end{array}$ & 0,48 & 1,59 & 4,71 & 7,76 & 2,10 & 1,41 \\
\hline $\begin{array}{l}\text { Farelo de soja } 3 \\
\text { Soybean meal } 3\end{array}$ & 0,50 & 1,59 & 4,73 & 7,63 & 2,02 & 1,38 \\
\hline $\begin{array}{l}\text { Farelo de soja } 4 \\
\text { Soybean meal } 4\end{array}$ & 0,47 & 1,58 & 4,93 & 8,11 & 2,08 & 1,52 \\
\hline $\begin{array}{l}\text { Farelo texturizado } \\
\text { Texturized soybean meal }\end{array}$ & 0,56 & 1,71 & 5,32 & 8,68 & 2,27 & 1,63 \\
\hline $\begin{array}{l}\text { Soja "Jet Sploder" } \\
\text { "Jet Sploder" soybean }\end{array}$ & 0,41 & 1,28 & 3,91 & 6,42 & 1,69 & 1,26 \\
\hline $\begin{array}{l}\text { Soja integral tostada } \\
\text { Toasted integral soybean }\end{array}$ & 0,43 & 1,44 & 4,11 & 6,78 & 1,75 & 1,28 \\
\hline $\begin{array}{l}\text { Soja micronizada } \\
\text { Micronized soybean }\end{array}$ & 0,45 & 1,42 & 4,13 & 6,81 & 1,72 & 1,32 \\
\hline
\end{tabular}

${ }^{1}$ Valores calculados através dos coeficientes de digestibilidade determinados in vivo e conteúdo de aminoácidos totais dos alimentos. ${ }^{2} \mathrm{CIS}$ (cistina), ALA (alanina), SER (serina), GLU (ácido glutâmico), ASP (ácido aspártico), TIR (tirosina).

1 Values calculated by the digestibility coefficients determined "in vivo" and total amino acids content of the feedstuffs.

${ }^{2}$ CYS (lisina), ALA (alanine), SER (serine), GLU (glutamic acid), ASP (aspartic acid), TYR (tyrosine).

Tabela 7 - Equações de predição do conteúdo de lisina (LIS), metionina (MET), metionina + cistina (M+C), treonina (TRE) e arginina (ARG) totais da soja e subprodutos, calculadas pela composição química dos alimentos

Table 7 - Prediction equations of total lysine (LYS), methionine (MET), methionine + cystine (Met + Cys), threonine (THR) and arginine (ARG) content of the soybean and by-products, calculated based on the chemical composition

\begin{tabular}{|c|c|c|c|c|c|c|}
\hline & $\begin{array}{c}\text { Constante } \\
\text { Constant }\end{array}$ & $\begin{array}{l}\mathrm{PB} \\
C P\end{array}$ & $\begin{array}{l}\mathrm{FB} \\
C F\end{array}$ & $\begin{array}{l}\mathrm{EE} \\
E E\end{array}$ & $\begin{array}{c}\mathrm{MM} \\
\text { Ash }\end{array}$ & $\mathrm{R}^{2}$ \\
\hline $\begin{array}{l}\text { LIS } \\
L Y S\end{array}$ & $-0,32161$ & 0,06731 & ---- & ---- & --- & 0,99 \\
\hline $\begin{array}{l}\text { MET } \\
M E T\end{array}$ & 0,02263 & 0,01323 & ---- & ---- & ---- & 0,96 \\
\hline $\begin{array}{l}\mathrm{M}+\mathrm{C} \\
\mathrm{Met}+\mathrm{Cys}\end{array}$ & 0,07894 & 0,02563 & ---- & ---- & --- & 0,94 \\
\hline $\begin{array}{l}\text { TRE } \\
\text { THR }\end{array}$ & 0,20930 & 0,03533 & ---- & ---- & ---- & 0,95 \\
\hline $\begin{array}{l}\text { ARG } \\
A R G\end{array}$ & $\begin{array}{l}-2,19880 \\
-0,39589\end{array}$ & $\begin{array}{l}0,11509 \\
0,08022\end{array}$ & ---- & $\begin{array}{c}0,01702 \\
---\end{array}$ & ---- & $\begin{array}{l}0,99 \\
0,98\end{array}$ \\
\hline & \multicolumn{6}{|c|}{$\begin{array}{l}\text { Correlações } \\
\text { Correlations }\end{array}$} \\
\hline $\begin{array}{l}\text { LIS } \\
L Y S\end{array}$ & ---- & 0,9935 & $-0,0393$ & $-0,9427$ & 0,8812 & ---- \\
\hline $\begin{array}{l}\text { MET } \\
\text { MET }\end{array}$ & --- & 0,9791 & 0,0357 & $-0,9518$ & 0,8877 & ---- \\
\hline $\begin{array}{l}\mathrm{M}+\mathrm{C} \\
M e t+C y s\end{array}$ & ---- & 0,9716 & $-0,0445$ & $-0,9472$ & 0,8606 & ---- \\
\hline $\begin{array}{l}\text { TRE } \\
\text { THR }\end{array}$ & --- & 0,9758 & $-0,0389$ & $-0,9362$ & 0,8414 & ---- \\
\hline $\begin{array}{l}\text { ARG } \\
A R G\end{array}$ & ---- & 0,9896 & $-0,0698$ & $-0,9094$ & 0,8691 & ---- \\
\hline
\end{tabular}


Tabela 8 - Equações de predição do conteúdo de lisina (LIS), metionina (MET), metionina + cistina (M+C), treonina (TRE) e arginina (ARG) digestíveis da soja e subprodutos, calculadas através da composição química

Table 8 - Prediction equations of digestible lysine (LYS), methionine (MET), methionine + cystine (Met + Cys), threonine (THR) and arginine (ARG) content of the soybean and by-products, calculated based on the chemical composition

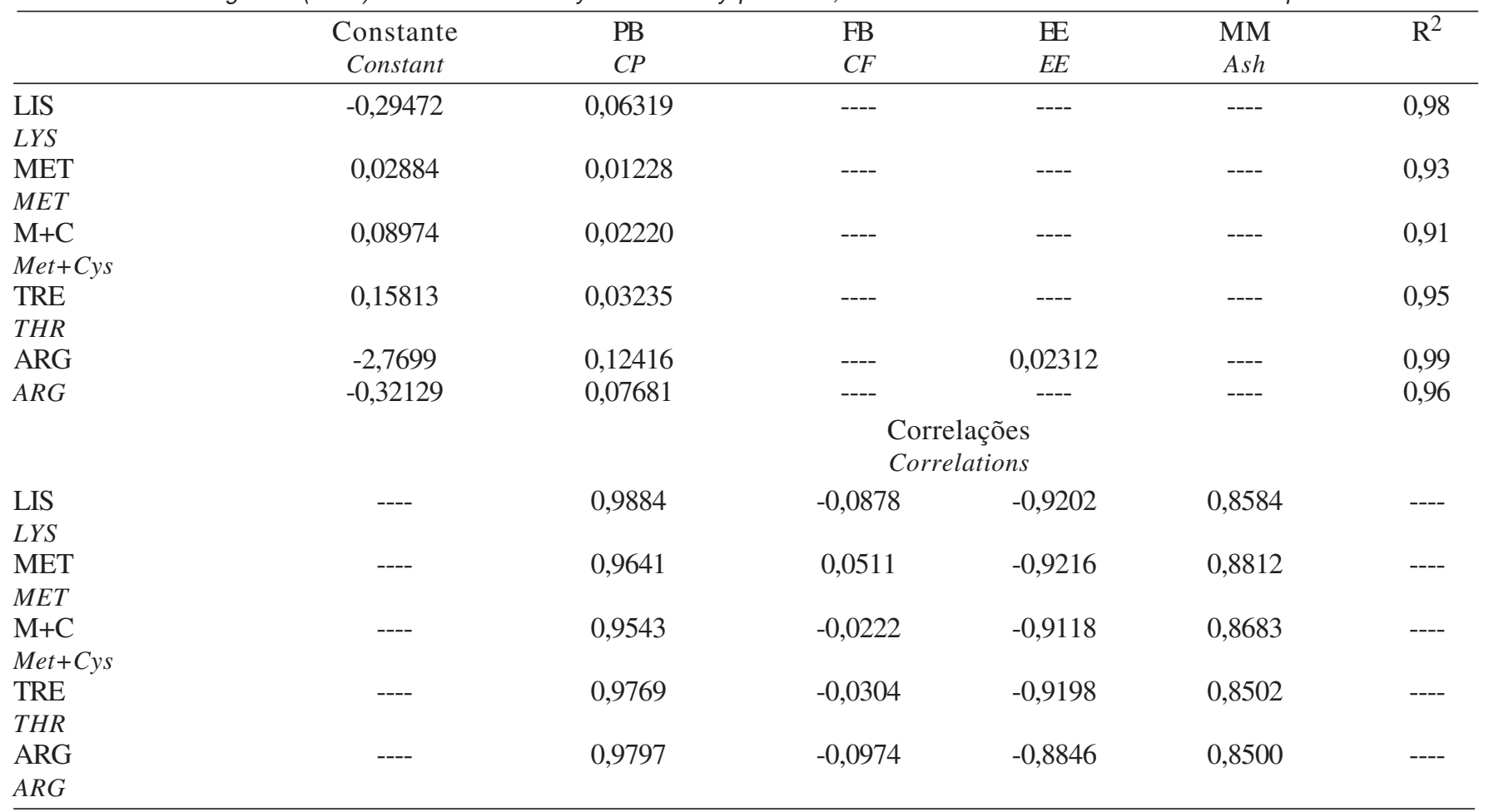

com valores superiores a $90 \%$ para os aminoácido totais e entre 88,19 (soma dos essenciais) e $95,18 \%$ (não essenciais). Estas correlações da PB com a lisina, treonina, metionina e metionina + cistina observadas no presente ensaio são maiores que aquelas apresentadas pelo NRC (1998), para os aminoácidos totais do farelo de soja, ressaltando-se que no presente trabalho as equações foram ajustadas através da composição de um grupo de alimentos e não de um alimento isoladamente. O NRC (1994) descreve equações para predizer a metionina, metionina + cistina, lisina e arginina do farelo de soja, contendo as variáveis $\mathrm{PB}$ e $\mathrm{MM}$, e equações com $\mathrm{PB}$, umidade, EE, FB e MM para predizer a treonina, estando em contradição ao ajustado no presente trabalho, no qual estas variáveis não compuseram a maioria dos modelos.

As estimativas dos conteúdos dos aminoácidos lisina, metionina, metionina + cistina, treonina e arginina totais e digestíveis dos alimentos estudados, preditas através das equações elaboradas, juntamente com os valores determinados no ensaio biológico e as médias da soma do quadrado dos desvios estão apresentadas nas Tabelas 9 e 10, respectivamente. Devido aos elevados valores de $\mathrm{R}^{2}$ obtidos no ajuste das equações com a PB, bem como sua alta correlação com os aminoácidos, foram consideradas nas estimativas as equações compostas com a variável PB apenas. Notase que os valores estimados foram bem semelhantes àqueles observados no ensaio in vivo, tanto para os aminoácidos totais quanto os digestíveis, o que pode ser comprovado através das médias da soma do quadrado dos desvios. Estas observações sugerem que as equações elaboradas podem ser seguras para estimar o conteúdo de aminoácidos totais e digestíveis deste grupo de alimentos, sendo de grande utilidade nas formulações de ração para aves. Entretanto, torna-se necessário novos trabalhos testando a aplicabilidade das equações em outras amostras destes alimentos e, possivelmente, fazendo determinações "in vivo" para comprovar a confiabilidade das estimativas. 
Tabela 9 - Estimativas do conteúdo de lisina, metionina, metionina + cistina (MET+CIS), treonina e arginina totais da soja e subprodutos, pelas equações de predição, em função da composição química dos alimentos (expressos com base na matéria seca) ${ }^{1}$

Table 9 - Estimates of total lysine, methionine, methionine plus cystine (Met+Cys), threonine and arginine contents of the soybean and by-products by the prediction equations, based on the chemical composition (data based in dry matter)

\begin{tabular}{|c|c|c|c|c|c|c|c|c|c|c|}
\hline \multirow[t]{2}{*}{$\begin{array}{l}\text { Alimento } \\
\text { Feedstuff }\end{array}$} & \multicolumn{2}{|c|}{$\begin{array}{l}\text { Lisina } \\
\text { Lysine }\end{array}$} & \multicolumn{2}{|c|}{$\begin{array}{l}\text { Metionina } \\
\text { Methionine }\end{array}$} & \multicolumn{2}{|c|}{$\begin{array}{c}\mathrm{MET}+\mathrm{CIS} \\
M e t+C y s\end{array}$} & \multicolumn{2}{|c|}{$\begin{array}{l}\text { Treonina } \\
\text { Threonine }\end{array}$} & \multicolumn{2}{|c|}{$\begin{array}{l}\text { Arginina } \\
\text { Arginine }\end{array}$} \\
\hline & $\mathrm{Obs}^{2}$ & $\mathrm{Est}^{3}$ & Obs & Est & Obs & Est & Obs & Est & Obs & Est \\
\hline $\begin{array}{l}\text { Farelo de soja } 1 \\
\text { Soybean meal } 1\end{array}$ & 3,04 & 3,01 & 0,68 & 0,68 & 1,38 & 1,35 & 1,98 & 1,96 & 3,54 & 3,57 \\
\hline $\begin{array}{l}\text { Farelo de soja } 2 \\
\text { Soybean meal } 2\end{array}$ & \multicolumn{10}{|c|}{ Soybean meal 2} \\
\hline $\begin{array}{l}\text { Farelo de soja } 3 \\
\text { Soybean meal } 3\end{array}$ & \multicolumn{4}{|c|}{ Soybean meal 3} & 1,33 & 1,35 & 1,90 & 1,96 & 3,56 & 3,59 \\
\hline $\begin{array}{l}\text { Farelo de soja } 4 \\
\text { Soybean meal } 4\end{array}$ & 3,17 & 3,18 & 0,69 & 0,71 & 1,35 & 1,41 & 2,01 & 2,05 & 3,82 & 3,77 \\
\hline \multicolumn{10}{|l|}{ Texturized soybean meal } & 3,83 \\
\hline \multicolumn{10}{|l|}{ "Jet Sploder" soybean } & 2,89 \\
\hline $\begin{array}{l}\text { Soja integral tostada } \\
\text { Toasted integral soybean }\end{array}$ & 2,48 & 2,45 & 0,58 & 0,57 & 1,15 & 1,13 & 1,70 & 1,66 & 2,97 & 2,90 \\
\hline \multicolumn{10}{|l|}{ Micronized soybean } & 2,91 \\
\hline $\begin{array}{l}\text { Médias das estimativas } \\
\text { Estimates means }\end{array}$ & 2,84 & 2,84 & 0,64 & 0,64 & 1,28 & 1,28 & 1,87 & 1,87 & 3,37 & 3,37 \\
\hline $\begin{array}{l}\text { Médias }{ }^{4} \\
\text { Means }^{4}\end{array}$ & \multicolumn{2}{|c|}{0,00130} & \multicolumn{2}{|c|}{0,00017} & \multicolumn{2}{|c|}{0,00086} & \multicolumn{2}{|c|}{0,00138} & \multicolumn{2}{|c|}{0,00299} \\
\hline $\begin{array}{l}{ }^{1} \text { Lisina }(\text { Lysine })=-0,32161+ \\
+0,02563 P B\left(R^{2}=0,94\right) \\
\left(R^{2}=0,98\right) . \\
{ }^{2} \text { Conteúdo de aminoácidos } \\
\text { galos adultos cecectomiza } \\
\text { cockerels). } \\
{ }^{3} \text { Valores estimados pelas e } \\
{ }^{4} \text { Médias da soma do quadra }\end{array}$ & $\begin{array}{l}1 \mathrm{~PB}(\mathrm{P} \\
\text { nina ( } t \\
\text { iveis v } \\
\text { igestibl }\end{array}$ & $\begin{array}{l}0,99 \\
\text { onine } \\
\text { ladei } \\
\text { mino }\end{array}$ & tidos & tern & $\begin{array}{l}2263 \\
\left(\mathrm{R}^{2}=\right. \\
\text { os co } \\
\text { igestit }\end{array}$ & $\begin{array}{l}1323 \\
\text {; }) \text { A } \\
\text { ntes }\end{array}$ & $\begin{array}{l}=0, \\
(A, \\
\text { estib } \\
\text { term }\end{array}$ & $\begin{array}{l}\mathrm{ET}+ \\
= \\
\text { e de } \\
\text { n vive }\end{array}$ & $\begin{array}{l}e t+C \\
89+ \\
\text { ados } \\
\text { cece }\end{array}$ & $\begin{array}{l}07894 \\
22 \mathrm{~PB} \\
\\
\text { o com } \\
\text { d adult }\end{array}$ \\
\hline
\end{tabular}


Tabela 10 - Estimativas do conteúdo de lisina, metionina, metionina + cistina $(\mathrm{M}+\mathrm{C})$, treonina e arginina digestíveis da soja e subprodutos, pelas equações de predição em função da composição química dos alimentos (expressos com base na matéria seca) ${ }^{1}$

Table 10 - Estimation of digestible lysine, methionine, methionine plus cystine (Met+Cys), threonine and arginine contents of the soybean and byproducts by the prediction equations based on the chemical composition (data based in dry matter)

\begin{tabular}{|c|c|c|c|c|c|c|c|c|c|c|}
\hline \multirow[t]{2}{*}{$\begin{array}{l}\text { Alimento } \\
\text { Feedstuffs }\end{array}$} & \multicolumn{2}{|c|}{$\begin{array}{l}\text { Lisina } \\
\text { Lysine }\end{array}$} & \multicolumn{2}{|c|}{$\begin{array}{l}\text { Metionina } \\
\text { Methionine }\end{array}$} & \multicolumn{2}{|c|}{$\begin{array}{c}\mathrm{MET}+\mathrm{CIS} \\
M e t+C y s\end{array}$} & \multicolumn{2}{|c|}{$\begin{array}{l}\text { Treonina } \\
\text { Threonine }\end{array}$} & \multicolumn{2}{|c|}{$\begin{array}{l}\text { Arginina } \\
\text { Arginine }\end{array}$} \\
\hline & $\mathrm{Obs}^{2}$ & $\mathrm{Est}^{3}$ & Obs & Est & Obs & Est & Obs & Est & Obs & Est \\
\hline $\begin{array}{l}\text { Farelo de soja } 1 \\
\text { Soybean meal } 1\end{array}$ & 2,85 & 2,83 & 0,64 & 0,64 & 1,22 & 1,19 & 1,77 & 1,76 & 3,43 & 3,48 \\
\hline $\begin{array}{l}\text { Farelo de soja } 2 \\
\text { Soybean meal } 2\end{array}$ & 2,77 & 2,78 & 0,64 & 0,63 & 1,17 & 1,17 & 1,76 & 1,73 & 3,35 & 3,41 \\
\hline $\begin{array}{l}\text { Farelo de soja } 3 \\
\text { Soybean meal } 3\end{array}$ & 2,78 & 2,84 & 0,64 & 0,64 & 1,19 & 1,19 & 1,71 & 1,76 & 3,47 & 3,49 \\
\hline $\begin{array}{l}\text { Farelo de soja } 4 \\
\text { Soybean meal } 4\end{array}$ & 2,98 & 2,99 & 0,65 & 0,67 & 1,19 & 1,24 & 1,84 & 1,84 & 3,72 & 3,67 \\
\hline $\begin{array}{l}\text { Farelo texturizado } \\
\text { Texturized soybean meal }\end{array}$ & 3,08 & 3,03 & 0,68 & 0,68 & 1,28 & 1,26 & 1,88 & 1,86 & 3,77 & 3,72 \\
\hline $\begin{array}{l}\text { Soja "Jet Sploder" } \\
\text { "Jet Sploder" soybean }\end{array}$ & 2,22 & 2,29 & 0,51 & 0,53 & 0,95 & 1,00 & 1,44 & 1,48 & 2,69 & 2,83 \\
\hline $\begin{array}{l}\text { Soja integral tostada } \\
\text { Toasted integral soybean }\end{array}$ & 2,35 & 2,30 & 0,56 & 0,53 & 1,04 & 1,00 & 1,54 & 1,49 & 2,92 & 2,84 \\
\hline $\begin{array}{l}\text { Soja micronizada } \\
\text { Micronized soybean }\end{array}$ & 2,35 & 2,31 & 0,52 & 0,54 & 1,01 & 1,00 & 1,49 & 1,49 & 2,93 & 2,94 \\
\hline Médias das estimativas & 2,67 & 2,67 & 0,61 & 0,61 & 1,13 & 1,13 & 1,68 & 1,68 & 3,29 & 3,28 \\
\hline $\begin{array}{l}\text { Médias } \\
\text { Means }^{4}\end{array}$ & \multicolumn{2}{|c|}{0,00208} & \multicolumn{2}{|c|}{0,00025} & \multicolumn{2}{|c|}{0,00107} & \multicolumn{2}{|c|}{0,00110} & \multicolumn{2}{|c|}{0,00546} \\
\hline
\end{tabular}

${ }^{1}$ Lisina $($ Lysine $)=-0,29472+0,06319 P B\left(R^{2}=0,98\right) ;$ Metionina (Methionine) $=0,02884+0,01228 P B\left(R^{2}=0,93\right) ;$ Metionina + cistina $($ Methionine + Cystine $)=0,08974+0,02220 \mathrm{~PB}\left(\mathrm{R}^{2}=0,91\right)$; Treonina (Threonine) $=0,15813+0,03235 \mathrm{~PB}\left(\mathrm{R}^{2}=0,95\right) ;$ Arginina (Arginine) $=-0,32129+0,07681 \mathrm{~PB}\left(\mathrm{R}^{2}=0,96\right)$.

2 Conteúdo de aminoácidos digestíveis verdadeiros obtidos por intermédio dos coeficientes de digestibilidade determinados in vivo com galos adultos cecectomizados (Digestible amino acids content obtained by the digestibility coefficients determined "in vivo" with cecectomized adult cockerels.

${ }^{3}$ Valores estimados pelas equações de predição (Estimated values by the prediction equations).

${ }^{4}$ Médias da soma do quadrado dos desvios (Square deviation sum means).

\section{Conclusões}

Os coeficientes médios de digestibilidade dos aminoácidos (essenciais e não essenciais, respectivamente) dos farelos de soja 1, 2, 3, 4 e texturizado, sojas integral Jet Sploder, integral tostada e micronizada foram 92,78 e 90,76; 92,66 e 89,70; 93,75 e 91,$26 ; 93,32$ e 90,$12 ; 91,55$ e 89,$08 ; 94,80$ e 92,34 ; 94,61 e 91,$47 ; 93,20$ e $90,47 \%$. As equações de predição foram ajustadas com altos valores de $\mathrm{R}^{2}$, explicando mais de $94 \%$ da variação dos aminoácidos totais e $91 \%$ dos digestíveis nos alimentos estudados, onde a proteína apresentou alta correlação positiva, fazendo boas estimativas do conteúdo em aminoácidos totais e digestíveis dos alimentos do grupo da soja; para predizer os aminoácidos totais e digestíveis da soja e subprodutos, podem ser utilizadas as equações LIS $=-0,32161+0,06731 P B\left(R^{2}=0,99\right) ;$ MET $=$

\section{R. Bras. Zootec., v.31, n.2, p.970-981, 2002 (suplemento)}

$0,02263+0,01323 \mathrm{~PB}\left(\mathrm{R}^{2}=0,96\right) ; \mathrm{M}+\mathrm{C}=0,078949$ $+0,02563 \mathrm{~PB}\left(\mathrm{R}^{2}=0,94\right) ; \mathrm{TRE}=0,20930+0,03533 \mathrm{~PB}$ $\left(\mathrm{R}^{2}=0,95\right) ; \mathrm{ARG}=-0,39589+0,08022 \mathrm{~PB}$ $\left(\mathrm{R}^{2}=0,98\right)$ e LIS $=-0,29472+0,06319 \mathrm{~PB}$ $\left(\mathrm{R}^{2}=0,98\right) ; \mathrm{MET}=0,02884+0,01228 \mathrm{~PB}\left(\mathrm{R}^{2}=0,93\right)$; $\mathrm{M}+\mathrm{C}=0,08974+0,0222 \mathrm{~PB}\left(\mathrm{R}^{2}=0,91\right) ;$ $\mathrm{TRE}=0,15813+0,03235 \mathrm{~PB}\left(\mathrm{R}^{2}=0,95\right)$ e $\mathrm{ARG}=$ $0,32129+0,07681 \mathrm{~PB}\left(\mathrm{R}^{2}=0,96\right)$, respectivamente.

\section{Literatura Citada}

ALBINO, L.F.T.; SILVA, M.A. Valores nutritivos de alimentos para aves e suínos determinados no Brasil. In: SIMPÓSIO INTERNACIONAL SOBRE EXIGÊNCIAS NUTRICIONAIS DE AVES E SUÍNOS, 1996, Viçosa, MG. Anais... Viçosa, MG: Universidade Federal de Viçosa, 1996. p.303-318.

ALBINO, L.T.F.; ROSTAGNO, H.S.; SANT'ANNA, R. et al. Determinação dos valores de aminoácidos metabolizáveis e proteína digestível de alimentos para aves. Revista da Sociedade Brasileira de Zootecnia, v.21, n.6, p.1059-1068, 1992. 
ALBINO, L.F.T.; COELHO, M.G.R.; RUTZ, F. et al. Valores energéticos e de triptofano de alguns alimentos determinados, em aves jovens e adultas. Pesquisa Agropecuária Brasileira, v.22, n.11/12, p.1301-1306, 1987.

ARABA, M.; DALE.; N.M. Evaluation of protein solubility as an indicator of overprocessing soybean meal. Poultry Science, v.69, n.1, p.76-83, 1990.

BELLAVER, C.; PARSONS, C.; EASTER, R.A. Estimativas da digestibilidade verdadeira de ingredientes de alimentos, obtida com galos cecotomizados em alimentação forçada. Pesquisa Agropecuária Brasileira, v.33, n.5, p.731-736, 1998.

DALE, N. Formulando com soya sobreprocesada. Indústria Avícola, v.44, n.3, 1997.

DALE, N. Ingredient analysis table. Feedstuffs, v.71, n.31, p.24-31, 1999.

DEGUSSA, A.G. Digestble amino acids in feedstuffs for poultry. Frankfurt, 1993, 18p.

EMPRESA BRASILEIRA DE PESQUISA AGROPECUÁRIA. EMBRAPA - CNPSA. Tabela de composição química e valores energéticos de alimentos para suínos e aves. 3.ed. CNPSA, 1991. 97p. (Documentos, 19).

FISCHER JR, A.A. Valores de energia metabolizável e de aminoácidos digestíveis de alguns alimentos para aves. Viçosa, MG: Universidade Federal de Viçosa, 1997. 55p. Dissertação (Mestrado em Zootecnia) - Universidade Federal de Viçosa, 1997.

FISCHER Jr, A.A.; ALBINO, L.F.T.; ROSTAGNO, H.S. et al. Determinação dos coeficientes de digestibilidade e dos valores de aminoácidos digestíveis de diferentes alimentos para aves. Revista da Sociedade Brasileira de Zootecnia, v.27, n.2, p.307-313, 1998.

NATIONAL RESEARCH COUNCIL - NRC. Nutrient requirements of swine. 3.ed. Washington, D.C.: National Academy Press, 1998. 189p.

NATIONAL RESEARCH COUNCIL - NRC. Nutrient requirements of poultry. 9.ed. Washington, D.C.: National Academy Press, 1994. 155p.

PUPA, J.M.R.; LEÃO, M.I.; CARVALHO, A.U. et al. Cecectomia em galos sob anestesia local e incisão abdominal. Arquivos Brasileiros de Medicina Veterinária e Zootecnia, v.50, n.5, p.531-535, 1998.
PUPA, J.M.R. Rações para frangos de corte formuladas com valores de aminoácidos digestíveis verdadeiros, determinados com galos cecectomizados. Viçosa, MG: Universidade Federal de Viçosa, 1995. 63p. Dissertação (Mestrado em Zootecnia) - Universidade Federal de Viçosa, 1995.

RHÔNE POULENC ANIMAL NUTRITION. Rhodimet ${ }^{\mathrm{TM}}$ Nutrition Guide. 2.ed. 1993. 55p.

RODRIGUES, P.B. Digestibilidade de nutrientes e valores energéticos de alguns alimentos para aves. Viçosa, MG: Universidade Federal de Viçosa, 2000. 204p. Tese (Doutorado em Zootecnia) - Universidade Federal de Viçosa, 2000.

ROSTAGNO, H.S.; SILVA, D.J.; COSTA, P.M.A. et al. Composição de alimentos e exigências nutricionais de aves e suínos (tabelas brasileiras). Viçosa, MG: Universidade Federal de Viçosa, 1983. 59p.

ROSTAGNO, H.S.; NASCIMENTO, A.H.; ALBINO, L.F.T. Aminoácidos totais e digestíveis para aves. In: SIMPÓSIO INTERNACIONAL SOBRE NUTRIÇÃO DE AVES, 1999, Campinas, SP. Anais... Campinas: Fundação Apinco de Ciência e Tecnologia Avícolas, 1999. p.65-83.

SIBBALD, I.R. A bioassay for available amino acids and true metabolizable energy in feedstuffs. Poultry Science, v.58, n.3, p.668-673, 1979.

SILVA, D.J. Análise de alimentos (métodos químicos e biológicos). 2.ed. Viçosa, MG: Universidade Federal de Viçosa, 1990. 165p.

UNIVERSIDADE FEDERAL DE VIÇOSA - UFV. SAEG Sistema de análises estatísticas e genéticas. Versão 5.0. Viçosa, MG: 1992. 59p. (Manual do usuário).

Recebido em: 18/09/01

Aceito em: 10/05/02 